\title{
Trichopsychodermatology: trichotillomania and trichophagia leading to Rapunzel syndrome
}

\author{
Krzysztof Balawender ${ }^{1}$, Anna Pliszka ${ }^{1}$, Klaudia Możdżeń ${ }^{1}$, Michał Kłos $^{1}$, Anna Ogorzałek ${ }^{1}$, Dariusz Boroñ ${ }^{2,3}$, \\ Beniamin O. Grabarek ${ }^{2,3}$
}

${ }^{1}$ Morphological Sciences Department of Human Anatomy, Institute of Medical Sciences, Medical College of Rzeszow University, Rzeszow, Poland

2Department of Histology, Cytophysiology and Embryology, Faculty of Medicine in Zabrze, University of Technology in Katowice, Poland ${ }^{3}$ Department of Gynecology and Obstetrics, Faculty of Medicine in Zabrze, University of Technology in Katowice, Poland

Adv Dermatol Allergol 2022; XXXIX (4): 739-743

DOI: https://doi.org/10.5114/ada.2021.112465

\begin{abstract}
Introduction: The Rapunzel syndrome occurs when the trichobezoar (hair ball) extends beyond the small intestine and sometimes even into the colon, producing long, tail-like hair extensions.

Aim: To present cases of trichobezoars, an extremely rare human intestinal disease caused by the ingestion of hair (trichophagia).

Material and methods: In this retrospective study, we assessed the medical records of 2 patients diagnosed with Rapunzel syndrome admitted to Academic Clinical Hospital No. 2 in Rzeszow, Poland.

Results: The first patient was a 15-year-old girl. The abdominal ultrasound examination revealed an abnormal, non-compressible structure with the approximate dimensions of $12 \times 11 \mathrm{~cm}$ in the epigastrium, with a strong shadow obscuring the view. Gastroscopy was performed and a tumour sample was taken for histopathological examination, which confirmed the diagnosis of a trichobezoar. The patient's mother did not agree to her daughter's psychiatric treatment. The second patient was a 15-year-old girl who reported to the Emergency Room in critical condition due to dehydration and long-term emesis with symptoms of cachexia. Ileotomy with the removal of two trichobezoars with a diameter of about $5 \mathrm{~cm}$ and $7 \mathrm{~cm}$ was performed. The patient was discharged for treatment at the Mental Health Clinic for trichotillomania.

Conclusions: Trichobezoars give non-specific symptoms that may imitate other diseases for example tumours. Psychotherapy is the recommended treatment and follow-up visits are important in preventing relapses.
\end{abstract}

Key words: Rapunzel syndrome, non-specific symptoms, diagnosis, psychiatric treatment.

\section{Introduction}

Trichotillomania is a psychiatric condition, defined as being a pathological, compulsive, self-induced, regular and repetitive hair-pulling, while trichophagia is eating previously torn out hair [1]. The occurrence frequency of trichotillomania is estimated to be $0.5-2.0 \%$, most recent studies found that $1.7 \%$ of people aged 16-69 suffer from it, with $30 \%$ of them suffering from trichophagia [2].

Accumulation of undigested foreign bodies in the gastrointestinal tract can lead to bezoar formation. We can differentiate them depending on the type of the main lodgement component, the most common examples are high-fibre plants (phytobezoars), pharmaceuticals (pharmacobezoars) and hair (trichobezoars). Clinicians can usually find them mainly in the stomach, among adolescent girls suffering from an eating disorder called pica. In adults, bezoar formation occurs more frequently due to gastroparesis, anatomical anomalies and gastric surgeries causing delay in stomach emptying and are more likely to accumulate indigestible foreign bodies [3]. Bezoars also appear in other parts of the digestive system, like the oesophagus, small and large intestine or even rectum [4].

Risk factors for bezoar formation are anatomical anomalies, partial gastrectomy, diabetes mellitus with gastroparesis, cystic fibrosis and certain psychiatric conditions.

Symptoms of the disease depend on the type of the bezoar. Pharmacobezoars may cause drug intoxication

Address for correspondence: Beniamin O. Grabarek PhD, Department of Histology, Cytophysiology and Embryology, Department of Gynecology and Obstetrics, Faculty of Medicine, University of Technology, Poland, e-mail: bgrabarek7@gmail.com Received: 2.10.2021, accepted: 27.10.2021. 
and symptoms related to the pharmaceutical effects, while phyto- and trichobezoars often lead to nausea, ulceration, epigastric pain, gastrointestinal bleeding and even perforation. Almost every bezoar is manifested through an obstruction and related symptoms such as constipation or diarrhoea, vomiting, recurrent, strong gastric pain, loss of appetite, anorexia, and weight loss [5, 6].

\section{Aim}

The aim of the study was to present trichobezoars, an extremely rare human intestinal disease caused by the ingestion of hair (trichophagia).

\section{Material and methods}

The work was registered on the website: www.researchregistry.com. The unique identifying number is: researchregistry7096. This study was conducted according to the guidelines of the Declaration of Helsinki. Due to the fact that this study is a retrospective analysis of data obtained in clinical routine care in an academic university setting, consent of the Ethics Committee was not required. According to the Polish statute, this is a non-interventional study (Article 37a (1) of the Pharmaceutical Law) and therefore, within the meaning of the Act on the professions of doctors and dentists of 5 December 1996, it does not require the Opinion of the Bioethical Committee and does not constitute a clinical trial. Informed consent was obtained from all subjects involved in the study. In this retrospective study, we assessed the medical records of 2 patients diagnosed with Rapunzel syndrome admitted to Academic Clinical Hospital No. 2 in Rzeszow.

Two cases of bezoars described in this study were trichobezoars, an extremely rare human intestinal disease caused by the ingestion of hair (trichophagia).

The first patient was a 15-year-old girl, who was admitted to academic clinical hospital no. 2 in Rzeszow; Paediatric Oncology Clinic in September 2018 because of a suspected stomach tumour detected by abdominal ultrasound.

The patient ignored weight loss and did not report any symptoms which could indicate an obstruction of the gastrointestinal tract. During a detailed physical examination, she admitted consuming only liquid foods. The girl confessed to paroxysmal abdominal pain for several months. For about 3 weeks she had been vomiting after solid foods. A few years earlier, she used to have behavioural disorders in the form of trichophagia associated with school stress, although according to the patient's mother, this symptom has not been present for 2 years. The results of the imaging tests and an extended interview with the patient's mother indicated that the trichobezoar could be the possible cause of the symptoms. The patient was transferred to the Paediatric Surgery Clinic where gastroscopy was performed, with a tumour sample taken for histopathological examination, which confirmed the diagnosis of trichobezoar in the stomach, the lumen of the pad and descending part of the duodenum. During the stay in the ward after gastroscopy, the following treatment was applied: electrolyte replacement; piperacillin + tazobactam; amikacin; esomeprazole; and fluconazole. Antibiotic and antifungal therapy was implemented due to yeast found in a bezoar swab.

The second patient was a 15-year-old girl who reported to the Emergency Room in critical condition due to dehydration and long-term emesis with symptoms of cachexia. After the diagnosis of acute kidney injury (AKI), she was admitted to academic clinical hospital no. 2 in Rzeszow; Paediatric Urology Clinic in July/August 2013.

The patient was moved to the Paediatric Surgery Clinic where the diagnostics were expanded to include further imaging tests.

During the stay in the ward, the following treatment was applied: electrolyte supplementation; ceftriaxone; and non-steroidal anti-inflammatory drugs (paracetamol, ibuprofen).

The patient was discharged in good condition a week after the surgery with a recommendation for treatment at the Mental Health Clinic for trichotillomania.

In both cases, the bezoar was removed by Artur Szymczak, a specialist paediatric surgeon. Both patients attended follow-up visits at the surgery clinic, and no further complications were found. We do not have knowledge if they started the psychiatric treatment.

\section{Results}

In the first patient, ultrasound revealed an abnormal, non-compressible structure with approximate dimensions of $12 \times 11 \mathrm{~cm}$ in the epigastrium with a strong shadow obscuring the view. Laboratory tests showed elevated procalcitonin (PCT), C-reactive protein (CRP) and the presence of erythrocytes in the urine. Chest $\mathrm{X}$-ray showed a visible shade under the left diaphragm dome with a cyclical outline and soft-tissue saturation of an ambiguous character. The abdominal ultrasound examination revealed an epigastric tumour mass described previously corresponding to a significantly enlarged stomach, filled with hyperechogenic structures which gave echo suppression behind the back wall - this picture might suggest a bezoar (Figure 1).

Similarly, echogenic structures filled with the lumen of the pad and descending part of the duodenum was noticed, the distal part was obscured by the distended stomach. Intestinal loops were pushed away with no oedema wall changes and no signs of widening. The gastrostomy with bezoar evacuation was performed (Figure 2).

The patient was discharged 11 days after the surgery, in good condition. The patient's mother did not agree to her daughter's psychiatric treatment. 
The abdominal ultrasound examination revealed gas bubbles in the portal vein trunk and distended stomach filled with fluid content. The gallbladder and common bile duct were invisible due to compression. Intestinal loops along the entire abdominal cavity were widened up to a maximum of $42 \mathrm{~mm}$ and were filled with fluid content. Peristalsis was intensified. Ultrasound revealed a gastrointestinal obstruction. Electrolyte disturbances detected in laboratory tests were corrected by obtaining normalization of urea and creatinine parameters. The computed tomography (CT) showed an obstruction of the jejunum and proximal ileum loops with oedema of the ileum wall up to $12 \mathrm{~mm}$. In the ileum of small pelvis, compact masses with dimensions of $7 \mathrm{~cm}$ and $5 \mathrm{~cm}$ were observed, with zero contrast enhancement suggesting bezoars (Figure 3).

Proximal obstruction of the small intestine was marked from these masses. Gastroscopy showed oesophageal abnormality along its entire length, it was congested and wallpapered with food residue. An abundant, green mucous lake was exposed in the stomach. Gastric mucosa was hyperaemic. On the upper wall of the duodenal bulb there was a large ulcer about $3 \mathrm{~cm}$ in diameter. The ileotomy with evacuation of two trichobezoars with a diameter of about 5 and $7 \mathrm{~cm}$ was performed.

\section{Discussion}

The syndrome is named after the long-haired Rapunzel from the Brothers Grimm fairy tale. Since 1968, there have only been 64 documented cases in the literature. This syndrome occurs when the trichobezoar (hair ball) extends beyond the small intestine and sometimes even into the colon, producing long, tail-like hair extensions. Rapunzel syndrome is characterized by a compulsive hair-pulling and swallowing disorder. There are several mental disorders associated with Rapunzel syndrome, such as trichophagia, trichotillomania, and pica. Trichophagia refers to someone who has a mental disorder and eats their own hair. Trichotillomania is the compulsion to pull out one's own hair, and most people begin to consume it after being pulled out. The syndrome itself is used to describe the manifestation of a trichobezoar that extends far into the small intestine. It describes the trichobezoar, not the underlying mental disorder. While this condition is extremely rare, scientists mention that it is absolutely crucial to follow prophylactic methods after surgery. The most common cause of relapse in patients is the lack of postoperative care and failure to complete psychological treatment. The researchers concluded that 92.5\% of all reported cases were successfully cured by laparotomy with a $99 \%$ success rate [7].

According to cases described above and other analysed cases, bezoars in X-rays are observed as a shade or mass usually visible when they are in stomach. In CT-scans

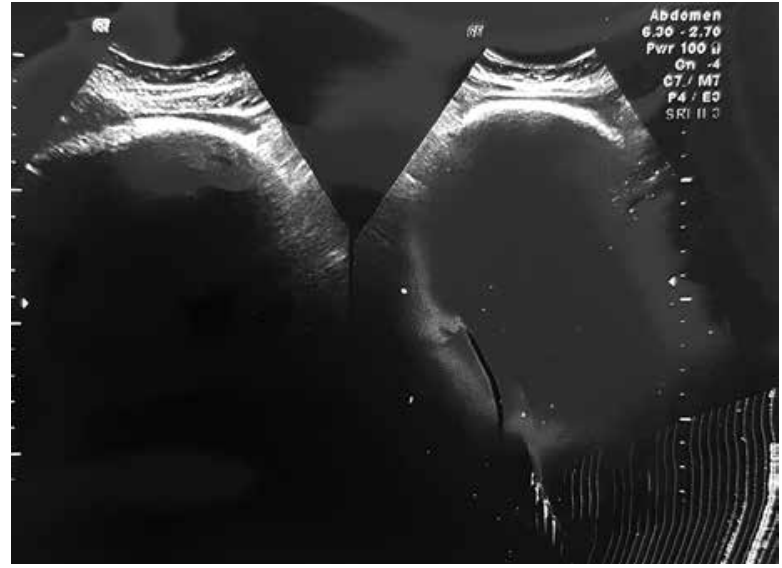

Figure 1. The abdominal ultrasound in patient 1

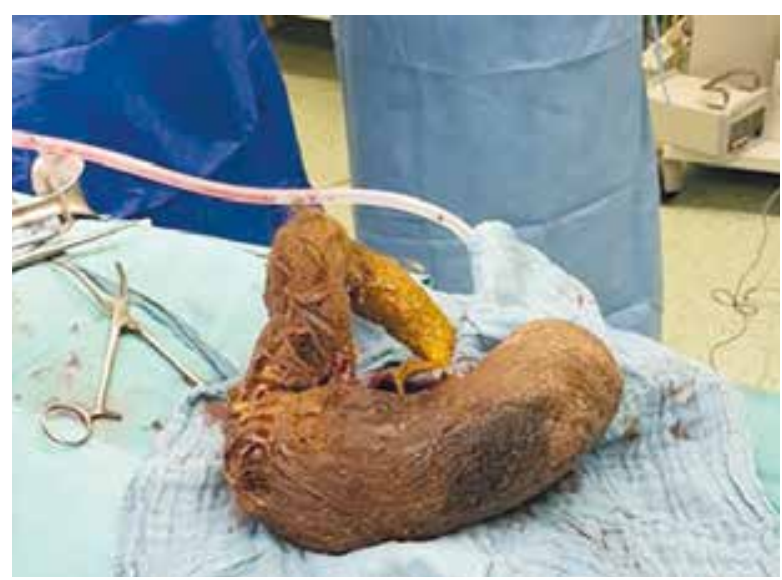

Figure 2. Bezoar in patient 1 evacuated during the surgery

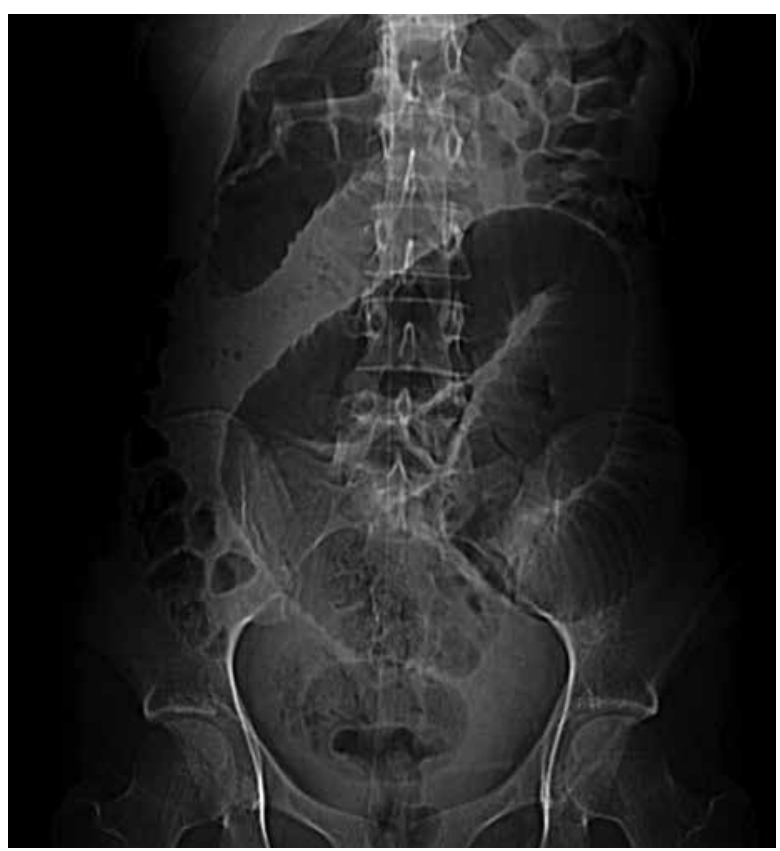

Figure 3. Results of the computed tomography performed in patient 2 
they appear as a round-shaped mass with air bubbles with obstruction of the gastrointestinal tract [8]. After analysis of all symptoms, X-rays and CT-scans, GI trichobezoars could initially be incorrectly diagnosed as a tumour or a cyst. That is why endoscopic examination is the most important in confirming or excluding the diagnosis of a bezoar, which can be confirmed by the presented cases - both patients were ultimately diagnosed through gastroscopy. Moreover, during the endoscopic procedure, a sample can be taken to confirm the diagnosis by histopathological examination. Undoubtedly, it is possible to make the correct diagnosis only with a CT-scan and even an X-ray, but again it depends on the type, size and location of the mass. The proper medical interview is significant in the diagnosis process because it may give us information or suggest the psychiatric disease (e.g. trichophagia). The goal of emergency treatment is the removal of the bezoar [5]. The treatment of trichobezoars consists of endoscopy, laparoscopy or laparotomy. The related success rates are: laparotomy - 100\%; laparoscopy - 75\%; and endoscopy 5\% [9]. Trichobezoars cannot be removed by dissolution, while such a method is used in cases of phytobezoars the therapeutic options are papain, acetylcysteine, cellulase, phosphoric acid and Coca-Cola [5].

For a trichobezoar to be created, the amount of hair consumed has to be very significant. It usually points to some psychiatric issues such as trichophagia and trichotillomania. Trichotillomania (TTM) is classified as a disorder of the obsessive-compulsive group along with excoriation disorder (skin picking disorder, or SPD) for example. [10]. It is one of body-focused repetitive behaviour (BFRB) disorders and there is a noticeable relation between TTM and anxiety disorders [11]. In this case hair-pulling and eating is basically a habit, therefore habit reversal training as part of behavioural therapy is very helpful [11]. In children, another management used for treating TTM is hypnotherapy, whereas in adults cognitive-behavioural therapy is the most common form of treatment. Alongside psychotherapy there are a few alternative forms of pharmacological approaches including SSRIs, tricyclic antidepressants and atypical neuroleptics, however, data are not fully consistent; further research will extend our understanding and improve management of the disease [12].

Although diagnosis of TTM can be difficult, we have some diagnostic tools that can be helpful: NIMH Trichotillomania Scale [9]; Yale-Brown Obsessive-Compulsive Scale-Trichotillomania (Y-BOCS-TM) [13]; and the Psychiatric Institute Trichotillomania Scale (PITS) [14].

Complications of $\mathrm{Gl}$ trichobezoars and its treatment might be malnutrition, anaemia, cachexia, gastric ulcer, or bowel obstruction. Furthermore, gastric perforation, pneumococcal peritonitis and coagulopathy were reported in some cases $[14,15]$.

Trichobezoars have non-specific symptoms that may imitate other diseases such as tumours. They are rare, but capable of resembling more common diseases. The symp- toms which can be worrisome for parents/guardians of the youngest patients are weight and/or appetite loss, sudden, strong stomach ache, bloody stool or hematemesis, and children are usually admitted to the emergency room in such a state. The psychiatric character of the disease is often negated by patients and their guardians, which contributes to difficulties in making the correct diagnosis. In summary, psychotherapy is the recommended treatment and follow-up visits are important in preventing relapses.

Psychotrichologic conditions are complex diseases that require a holistic view of the patient and the provision of interdisciplinary help.

\section{Acknowledgments}

We would like to thank for improving and supporting our work and Mr Oskar Ogloszka for checking our English.

\section{Conflict of interest}

The authors declare no conflict of interest.

\section{References}

1. Grant JE, Chamberlain SR. Trichotillomania. Am J Psychiatry 2016; 173: 868-74.

2. Grover S, Mehra A, Avasthi A, Gupta V. Trichophagia along with trichobezoar in the absence of trichotillomania. J Neurosci Rural Pract 2014; 5 (Suppl 1): 55-57.

3. Samiullah K, Kui J, Lan-ping Z, et al. Upper gastrointestinal manifestation of bezoars and the etiological factors: a literature review. Gastroenterol Res Pract 2019; 2019: 5698532.

4. Manatakis D, Sioula M, Passas I, et al. Rectal seed bezoar due to sunflower seed: a case report and review of the literature. Pan Afr Med J 2018; 31: 157.

5. Eng K, Kay M. Gastrointestinal bezoars: history and current treatment paradigms. Gastroenterol Hepatol 2012; 8: 776-78.

6. Tayyem R, llyas I, Smith I, Pickford I. Rapunzel syndrome and gastric perforation. Ann R Coll Surg Engl 2010; 92: 27-8.

7. Godlewska J, Orpiszewska J, Górecki W. Nawrotowy trichobezoar u pacjentki z zespołem Roszpunki. Pediatr Med Rodz 2021; 17: 64-6.

8. Iwamuro M, Okada H, Matsueda K, et al. Review of the diagnosis and management of gastrointestinal bezoars. World J Gastrointest Endosc 2015; 7: 336-45.

9. Swedo SE, Rapoport JL, Leonard H, et al. Obsessive-compulsive disorder in children and adolescents: clinical phenomenology of 70 consecutive cases. Arch Gen Psychiatry 1989; 46: 335-41.

10. Henkel ED, Jaquez SD, Diaz LZ. Pediatric trichotillomania: review of management. Pediatr Dermatol 2019; 36: 803-7.

11. Grant JE, Chamberlain SR. Trichotillomania. Am J Psychiatry 2016; 173: 868-74.

12. Woods DW, Houghton DC. Diagnosis, evaluation, and management of trichotillomania. Psychiatr Clin North Am 2014; 37: 301-17.

13. Stanley TR, Burnham KP. A closure test for time-specific capture-recapture data. Environ Ecol Stat 1999; 6: 197-209. 
14. Jafferany M, Patel A. Trichopsychodermatology: the psychiatric and psychosocial aspects of hair disorders. Derm Ther 2020; 33: e13168.

15. Hon KL, Cheng J, Chow CM, et al. Complications of bezoar in children: what is new? Case Rep Pediatr 2013; 2013: 523569. 